kleine Dinocephalen sind bisher auch nicht bekannt. Eine gründliche Umprägung der Außenelemente des Skelettes würde zwischen beiden noch nötig sein. Der erste Teil der Vergleichung ergibt, daß3 die Sauropterygier sich in die verschiedenen Gruppen frühzeitig verzweigen, auch die Plesiosaurier setzen früh an.

Tübingen, den 21. April 1920.

\title{
Systematische und genetische Betrachtungen über die Stegocephalen.
}

\author{
Von Friedrich von Hnene in Tübingen.
}

(Eingegangen am 27. April 1920.)

Durch Zittel ist die Einteilung der Stegocephalen (sensu lat.) nach der Wirbelform eingeführt worden. Sie hat sich bis in die letzte Zeit durchaus behauptet. Der grundlegende Gedanke hiervon ist entschieden ausgezeichnet und hat ja auch tatsächlich die anderen Einteilungsprinzipien verdrängt. Aber trotz dieser allgemeinen Anerkennung der leitenden Idee in rein systematischer Hinsicht ist sie doch nicht ernst genug genommen worden, denn auf genetischem Gebiet ist sie nicht mit der nötigen Konsequenz angewendet worden. Nachdem Gadow 1896 auf embryologischem Wege die Morphogenie der Wirbel kennen gelehrt hatte, stand der Weg zu ihrer Anwendung auf die Stammesgeschichte offen. Nichtsdestoweniger konnte man bis in allerletzte Zeit auch von kenntnisreichen Paläontologen den Gedanken einer Abstammung der Reptilien resp. der Cotylosaurier von den temnospondylen Stegocephalen ausgesprochen oder angedeutet finden. Verf. hat erstmals 1913 (Abschnitt "Stegocephalen“ im Handwörterbuch für Naturwissenschaften, Bd. 9. S. 503 und 508) darauf aufmerksam gemacht, daß infolge des so verschiedenartigen Aufbaus der Wirbel bei den verschiedenen Zweigen der Stegocephalen und Amphibien und den Reptilien und Säugetieren diese zum großen Teil nicht genetisch aufeinander zuriickgeführt werden können, sondern als parallel oder von einer gemeinsamen Stelle divergierend aufgefaßt werden müssen. Diesen damals nur kurz ausgesprochenen Gedanken, dessen weitere Ausführung und volle Anwendung ich mir auf die Sommerferien 1914 aufgespart hatte (die dann durch den Krieg unmöglich gemacht wurde), hat zu meiner großen Freude und Genugtuung O. Abel in seinem 1919 erschienenen Buch „Die Stämme der Wirbeltiere" aufgegriffen und, so wie er gemeint war, in mustergültiger Weise ausgeführt. Ich möchte nur vielleicht noch etwas stärker die phylogenetische Bedeutung betonen. 
Die sechs verschiedenen Wirbeltypen sind kurz (z. T. mit Abels Worten) folgende:

1. Rhachitom. Aus allen vier Paaren von Elementen aufgebaut (Basiventralia $=$ Hypocentrum $;$ Basidorsalia $=$ Neuralbogen; Interventralia $=$ Intercentrum pleurale; Interdorsalia $=$ Pleurocentrum). Sie bleiben entweder getrennt oder verschmelzen teilweise. Den Hauptanteil haben die Basiventralia. Interventralia klein oder verloren.

2. Embolomer. Basidorsalia + Basiventralia und Interdorsalia + Interventralia bilden je einen gleich großen Wirbel, so daß ein „Doppelwirbel" entsteht. Die vordere Scheibe bildet den "Hauptwirbel" mit Neuralbogen und Rippenansatz, die hintere den "Zwischenwirbel".

3. Stereospondyl. Die Basiventralia bilden die Hauptmasse des Wirbelkörpers und die Basidorsalia den oberen Bogen, Interventralia knorpelig und Interdorsalia verloren.

4. Pseudocentral. Hauptmasse des Wirbelkörpers aus den Basidorsalia gebildet. Basiventralia zurücktretend. Beide andern Elemente knorpelig.

5. Notocentral. Die Hauptmasse des Wirbelkörpers bilden die Interdorsalia, Interventralia mit ihnen verschmolzen oder verdrängt. Basiventralia mit den Basidorsalia verschmolzen oder verdrängt.

6. Gastrocentral. Hauptmasse des Wirbelkörpers von den Interventralia gebildet. Die Basiventralia nehmen nur als Intercentra und Hämapophysen teilweisen Anteil am Wirbelbau. Interdorsalia verloren.

Der embolomere und der stereospondyle Wirbelbau kann als besonderer Fall des rhachitomen, resp. als von ihm ausgehend betrachtet werden. Es bleiben also vier Grundfälle: 1. der rhachitome, 2. der pseudozentrale, 3. der notocentrale, 4. der gastrocentrale. Diese sind faktisch und historisch als die vier ursprünglichsten Radiationen der tetrapoden Wirbeltiere aufzufassen. Sie sind einander völlig gleichwertig und Glieder eines dieser vier Stämme können unmöglich von Gliedern eines der andern abstammend gedacht werden, sondern sie konvergieren rückwärts gegeneinander.

Die phylogenetische Wichtigkeit der Wirbelbildung kann kaum überschätzt werden. Vielleicht habe ich bald Gelegenheit, ausführlicher darauf zurückzukommen.

Die zu den obigen Gruppen gehörigen Tiere gruppieren sich in starker Anlehnung an Abels Ausführungen, jedoch mit einigen Abweichungen wie folgt. Dabei bedeuten die mit römischen Ziffern bezeichneten Gruppen die vier Grundstämme. Die hier aus technisch-praktischen Gründen beibehaltene Klassifizierung in Unterordnungen, Ordnungen, Unterklassen und Klassen ist hier ohne irgendwelche phylogenetische Bedeutung, sondern nur als Hergebrachtes verwendet. 
I. Rhachitomi

Embolomeri

Stereospondyli

Chauliodontidà $e$

Labyrinthodontidae

Plagiosauridae

II. Pseudocentrophori Nectridae

Ptyonidae

Aistopodidae

Phyllospondyli

Lysorophidae

Urodela

Apoda

III. Anura $=$ Notocentrophori

IV. (Gastrocentrophori) Microsauria
Archegosauridae

Trimerorhachidae

Eryopinae

Trematopsidae

Dissorophidae

Aspidosauridae

Zatrachyidae

Micropholidae

Cricotidae

$\begin{array}{ll} & \text { Ophiderpetontidae } \\ & \text { Molgophidae } \\ & \text { Phlegetontidae } \\ \text { Phyllospondyli } & \text { Branchiosauridae } \\ \text { Lysorophidae } & \text { Acanthostomidae }\end{array}$

Cotylosauria und durch sie alle andern Reptilien, die Vögel und die Säugetiere.

Der faktische Wert des grundlegenden Wirbelbaues ist deshalb so viel größer als alle auf Schädeldeckknochen, Gürtel- und Extremitätenskelett gegründeten Unterscheidungsmerkmale, weil es sich hier um die ersten Schutzelemente des zuerst angelegten Neutralrohres mit der Chorda dorsalis handelt. Es spiegeln sich hierin also die ersten Radiationsneigungen des Wirbeltierstammes. Alle andern phylogenetisch verwertbaren Teilungsprinzipien sind diesem untergeordnet. Hier ist auch in erster Linie die Stelle, von der aus auf die Fische und ihr Verhältnis zu den Tetrapoden geblickt werden könnte; die Extremitäten, mit denen man es schon versucht hat, eignen sich dazu nicht. Nur Tetrapodenformen, die in der Zusammensetzung der Wirbel die Merkmale mehrerer oder aller der vier Hauptstämme vereinigen, können Anspruch erheben, den zu postulierenden primitivsten Wirbeltieren anzugehören, aus denen jene hervorgegangen sind. Das Verhältnis zu den Vertretern dieser Stämme kann ein analoges sein wie das der Embolomeri und 
der Stereospondyli zu den Rhachitomi. Man kann solches nur im Karbon oder wahrscheinlich früher erwarten. -

Da bei den gegenwärtigen Zuständen der Druck sehr langsam geht, will ich hier auch kurz über die systematischen Ergebnisse einer in der Presse befindlichen Arbeit über Gonioglyptus; einen alttriassischen Stegocephalen aus Indien berichten. Der dort beschriebene Schädel einer neuen Art stammt aus den untersten ammonitenreichen Triaskalken von Chideru in der Salt Range Im vergleichenden Teil habe ich mich der genaueren Verwandtschaft der Gattungen der Unterordnung Labyrinthodontidae zugewandt und bin zu der folgenden Gruppierung gelangt:

1. Fam. Trematosauridae fam. nov.

Genus Trematosaurus Braun

Tertrema Wiman

Platystega Wiman

Lyrocephalus Wiman

Aphaneramma A. S. Woodward

Gonioglyptus Huxley

Lonchorhynchus Wiman

? Glyptognathus Lydekker

2. Fam. Mastodonsauriḋae fam. nov.

Mastodonsaurus Jaeger

Labyrinthodon Owen

Diadetognathus Miall

Pachygonia Huxley

3. Fam. Capitosauridae fam. nov.

Capitosaurus H. v. Meyer

Cyclotosaurus E. Fraas

4. Fam. Metoposauridae fam. nov.

Metopias H. v. Meyer

Anaschisma Branson

Dictyocephalus Leidy

5. Fam. Rhytidosteidae fam. nov.

Rhytidosteus Owen

Nur drei unsichere Gattungen bleiben übrig: Eupelor gehört vielleicht zur zweiten oder dritten Familie. Pariostegus ist wahrscheinlich überhaupt kein Labyrinthodontide. Hercynosaurus kann vielleicht zur zweiten Familie gehören. Diese fünf Familien sind unter sich streng gesonderte Gruppen.

Tübingen, den 20. April 1920. 